\title{
ATRATIVIDADE DE ISCAS ALIMENTARES NA CAPTURA DE INSETOS EM FLORESTA NATIVA E SISTEMA AGROFLORESTAL
}

\author{
Adeissany Stephany Ramos Machado dos Santos ${ }^{1}$ \\ Mirian Gusmão \\ Thiago Luíz Mauro ${ }^{3}$ \\ Ritielly Laiany Carvalho Senigalia ${ }^{4}$ \\ Elisangela Clarete Camili ${ }^{5}$
}

\section{INTRODUÇÃO}

Cada vez mais o país adota sistemas de manejo que integram lavoura, pecuária e florestas, pois, representam uma opção de manejo sustentável do solo que pode gerar produtos para comercialização no mercado e de subsistência familiar. Estes sistemas, proporcionam diversidade vegetal e proteção ao solo, pois, seus resíduos servem de alimento aos animais edáficos. Portanto, em sistemas agroflorestais (SAF) a população e a diversidade da fauna do solo tende a aumentar em comparação com sistemas de cultivo mais intensivos e menos diversificados como os agrícolas (BROWN et al., 2009).

As pertubações causadas por ações antrópicas podem ser monitoradas por meio dos padrões de mudança na biodiversidade e, algumas espécies de insetos, ou mesmo grupo de espécies, atuam como bioindicadores da degradação ambiental (SANTOS et al.,

1 Mestranda do Programa de Pós-Graduação em Ciências Florestais e Ambientais, Faculdade de Engenharia Florestal - UFMT, Cuiabá, MT, Brasil - Adeissany@gmail.com.

2 Mestranda do Programa de Pós-Graduação em Ciências Ambientais, Departamento de Engenharia Florestal, Universidade Federal de Rondônia - UNIR, Campus Rolim de Moura, RO.

3 Graduando no curso de Engenharia Florestal pela Universidade Federal de Rondônia - UNIR, Campus Rolim de Moura, RO.

4 Mestranda do Programa de Pós- Graduação em Agricultura Tropical, Departamento de Fitotecnia e Fitossanidade, Faculdade de Agronomia e Zootecnia, FAAZ - UFMT, Cuiabá, MT, Brasil

5 Professora Dra. Associada, Departamento de Fitotecnia e Fitossanidade, Faculdade de Agronomia e Zootecnia, FAAZ - UFMT, Cuiabá, MT, Brasil. 
2006), um parâmetro para a avaliação do impacto é a presença ou a ausência de determinadas populações (RÉ, 2007).

As formigas são consideradas como bioindicadoras, pois, apresentam grande diversidade de espécies e são ecologicamente importantes na decomposição da serapilheira em florestas tropicais (AGOSTI et al., 2000).

Armadilhas abastecidas com iscas alimentares são utilizadas na captura de insetos há décadas. Tais iscas, são compostas à base de frutas e de hidrolisado de proteína de milho e, proporcionam redução dos custos e do impacto ambiental comparadas a iscas artificiais (MEDEIROS, 2009).

$\mathrm{O}$ abastecimento de armadilhas com atrativos alimentares à base de frutas tais como suco de pêssego, abacaxi, laranja, acerola, maracujá, goiaba, uva, manga e de mamão são constantemente utilizados (VELOSO et al., 1994; SALLES, 1999; CORNELIUS et al., 2000; LEMOS et al., 2002; CASTREJON-GÓMEZ et al., 2004; BHARATHI et al., 2004; MONTES e RAGA, 2006).

Atrativos alimentares são importantes, pois, estão relacionados com a reprodução dos insetos, visto que as fêmeas necessitam de compostos protéicos para atingirem a maturidade sexual (NAKANO et al., 1981; PEREIRA, 2001; GARCIA-RAMIREZ et al., 2004).

Estudos em florestas nativas no Brasil ainda são poucos, os trabalhos existentes são em povoamentos exóticos, onde os insetos são considerados como praga (PELENTIR, 2007). De igual maneira, são poucos os trabalhos desenvolvidos sobre as comunidades de animais edáficos em SAF brasileiros, principalmente os que comparam as populações encontradas em SAF com sistemas naturais, como florestas em processo de regeneração (BROWN et al., 2009).

Desta forma, este trabalho tem por objetivo avaliar a atratividade de iscas alimentares sobre algumas ordens de insetos entre uma área de floresta nativa e de SAF, correlacionando com as características de cada ambiente.

\section{DELINEAMENTO EXPERIMENTAL}

As áreas de estudo situam-se na região Centro-Sul do estado de Rondônia, no município de Rolim de Moura (Figura 1). O fragmento de floresta nativa está localizado na Linha 180 Sul km 8, 
nas coordenadas $11^{\circ} 47^{\prime} 29,97^{\prime \prime} \mathrm{S}$ e $61^{\circ} 48^{\prime} 54,02^{\prime \prime} \mathrm{W}$ com altitude de $253 \mathrm{~m}$, e a área do SAF situa-se na Linha 172 Norte km 15 , nas coordenadas $11^{\circ} 36^{\prime} 11,37^{\prime \prime} \mathrm{S}$ e $61^{\circ} 53^{\prime} 8,95^{\prime \prime} \mathrm{W}$ com altitude de $210 \mathrm{~m}$. No momento do desenvolvimento desta pesquisa, o Sistema Agroflorestal tinha 25 anos de implantação, onde o cupuaçu foi introduzido em um fragmento de floresta sem espaçamento padronizado e, os tratos culturais são bastante escassos favorecendo a regeneração natural.

O clima apresenta homogeneidade espacial e sazonal da temperatura média do ar, e uma variabilidade temporal em relação à precipitação pluviométrica (SIPAM, 2006). Segundo a classificação de Köppen, o clima é do tipo Am-Tropical com temperatura que varia de 24 a 26 @C, precipitação anual de 2.200 a $2.500 \mathrm{~mm}$ e umidade relativa do ar oscilando em torno de $85 \%$ no período chuvoso, entre outubro e maio (MARIALVA, 1999; ALVARES et al., 2013). A tipologia estudada predominante compreende a Floresta Ombrófila Aberta Submontana (IBGE, 2012).

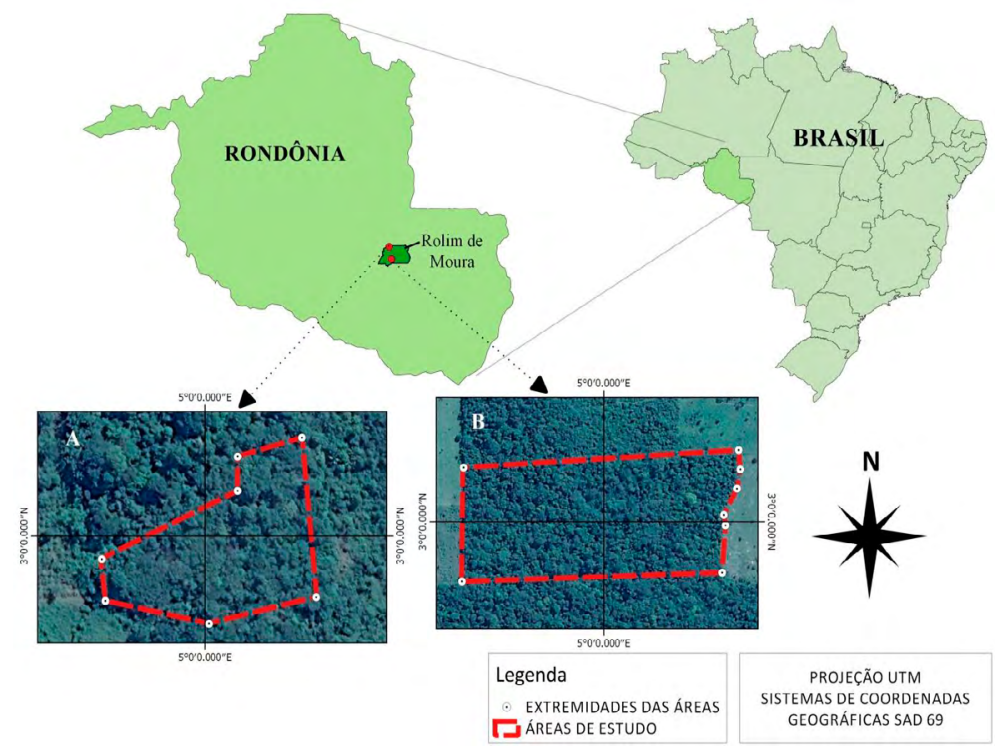

Figura 1. Localização das áreas de estudo, Rolim de Moura - RO, 2014. (A) Sistema Agroflorestal; (B) Fragmento de Floresta Nativa

Fonte: IBGE, 2012. 
Em cada área de estudo foi demarcada uma parcela de 50×30 m onde foram delimitadas e sorteadas cinco subparcelas de 10x10 m (Figura 2). O SAF escolhido apresenta composição florística bem próxima à floresta nativa regional, indicando equilíbrio entre sustentabilidade e rentabilidade.
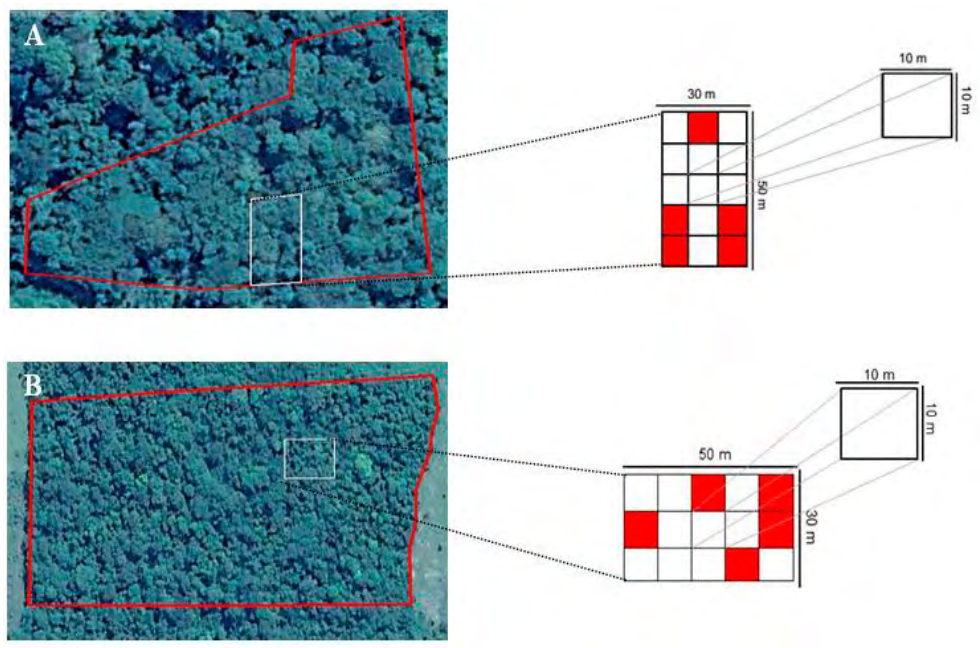

Figura 2. Representação das parcelas e subparcelas das áreas de estudo localizadas no município de Rolim de Moura/RO, 2014. (A) Fragmento de floresta nativa, denominado Sítio Bárbara Vitória. (B) SAF, denominado Sítio Dois Irmãos.

Com o intuito de atrair os insetos, em cada subparcela foram instaladas iscas de sardinha com paçoca e mamão com manga, sobre recipiente de plástico, a escolha das iscas foram adaptações dos trabalhos de Medeiros (2009), Simões (2009), Oliveira et al. (2013) e, Silva et al. (2014). A coleta foi realizada no período matutino, contabilizando 30 minutos a partir da observação do contato do primeiro inseto com a isca. Foi utilizado pinças e redes entomológicas (para insetos voadores) na captura. Os insetos capturados foram acondicionados em recipientes devidamente identificados com o número da subparcela e, armazenados em álcool 70\% (no caso de insetos pequenos $<1 \mathrm{~mm}$, como mosquitos) ou congelados (no caso de insetos maiores). 


\section{RESULTADOS E DISCUSSÃO}

A classificação dos insetos capturados nos dois ambientes com as duas iscas (Tabela 1) mostra diferença de incidência quanto aos ambientes e iscas. Na floresta nativa houve maior diversidade de insetos na isca com frutas. Já no SAF, apareceram as mesmas famílias nas duas iscas.

Dentre os grupos encontrados nesse tipo de estudo, destacam-se a presença das ordens Coleoptera, Hymenoptera e Orthoptera. A ordem Coleoptera é a mais numerosa entre os insetos e apresenta uma grande diversidade tanto ecológica quanto morfológica (RUPPERT et al., 2005), sendo muitos de seus integrantes tipicamente associados a material em decomposição. A ordem Hymenoptera é bastante conhecida pelo seu papel na polinização, no entanto carcaças são consideradas importantes fontes alimentares para vespas sociais e abelhas da região neotropical (SILVEIRA et al., 2005). Algo similar pode ser observado para ordem Orthoptera, que é amplamente conhecida por seu hábito fitófago, mas algumas famílias podem se alimentar de outros insetos (TRIPLEHORN; JOHNSON; 2005).

\begin{tabular}{ccc}
\hline \multirow{2}{*}{ ISCAS } & \multicolumn{2}{c}{ Floresta Nativa } \\
\hline & Ordem & Família \\
\cline { 2 - 3 } MAMÃO + MANGA & Coleoptera & Chrysomelidae \\
\cline { 2 - 3 } & Diptera & Oestridae \\
\cline { 2 - 3 } & Hymenoptera & Formicidae \\
\hline \multirow{2}{*}{ SARDINHA + PAÇOCA } & Orthoptera & Acrididae \\
& Coleoptera & Chrysomelidae \\
& Diptera & Oestridae \\
\hline & Hymenoptera & Formicidae \\
\hline MAMÃO + MANGA & & Oestridae \\
& Diptera & Vespidae \\
& Hymenoptera & Formicidae \\
& Hymenoptera & Acrididae \\
\hline
\end{tabular}

Continua... 


\begin{tabular}{lcc}
\hline & Diptera & Oestridae \\
SARDINHA + PAÇOCA & Hymenoptera & Vespidae \\
& Hymenoptera & Formicidae \\
& Orthoptera & Acrididae \\
\hline
\end{tabular}

Tabela 1. Classificação dos insetos capturados através de iscas alimentares em Floresta Nativa e Sistema Agroflorestal, Rolim de Moura - RO, 2014

Há, aproximadamente, 950 mil espécies de insetos formalmente descritas em todo o mundo (LEWINSOHN; PRADO, 2005). Os membros da classe Insecta são arranjados em 29 ordens (GRIMALDI; ENGEL, 2005). Quatro dessas ordens, Coleoptera, Diptera, Hymenoptera e Lepidoptera somam $81 \%$ de todas as espécies de insetos descritas até o momento. A grande diversidade de espécies, junto com a ampla gama de hábitos alimentares e, ainda, a vasta distribuição, os torna de grande importância ecológica e econômica.

As espécies das ordens Coleoptera, Diptera, Hymenoptera e Orthoptera são considerados constituintes da macrofauna e a sua abundância pode alcançar vários milhões de indivíduos por hectare e sua biomassa várias toneladas por hectare (BROWN et al., 2001). Sua diversidade também pode superar 1.000 espécies por local em ecossistemas altamente diversos como as florestas tropicais, ainda que existam pouquíssimos dados sobre a diversidade total da macrofauna edáfica em locais específicos na região tropical (BARROS et al., 2006).

Simões et al. (2009) avaliaram iscas de suco de maracujá com caldo de sardinha e verificaram que o uso de armadilhas iscadas é uma importante ferramenta na avaliação da abundância e distribuição de ordens de insetos em ambientes naturais. Entretanto as mesmas são seletivas em relação à composição e quantificação dos táxons. A análise de variância (Tabela 2) demonstra que não houve diferença na incidência dos insetos nos ambientes de floresta nativa e sistema agroflorestal e suas interações.

\begin{tabular}{cccc}
\hline FV & SQ & QM & F \\
\hline Iscas & 55,12 & 55,12 & $110,30 * *$ \\
Ambientes & 0,12 & 0,12 & $0,25 \mathrm{~ns}$ \\
Ordens & 331,4 & 110,45 & $220,90 * *$
\end{tabular}

Continua... 


\begin{tabular}{cccc} 
Iscas x Ambientes & 36,12 & 36,12 & $72,25 * *$ \\
Iscas x Ordens & 169,37 & 56,45 & $112,90^{* *}$ \\
Ambientes x Ordens & 42,37 & 14,12 & $28,25^{* *}$ \\
Iscas x Ambientes x Ordens & 124,37 & 41,45 & $82,90 * *$ \\
\hline CV & $18,55 \%$ \\
\hline
\end{tabular}

Tabela 2. Análise de variância para a variável número de insetos coletados através do métodos de iscas alimentares nos ambientes de Floresta Nativa e Sistema Agroflorestal, Rolim de Moura - RO, 2014

** = significativo ao nível de $1 \%$ de probabilidade $(p<0,01)$

$\mathrm{ns}=$ não significativo $(\mathrm{p}>=0,05)$

A média dos tratamentos (Tabela 3) mostra que das iscas utilizadas, a que apresentou maior atratividade para os insetos foi mamão com manga. Em trabalho realizado por Medeiros (2009) quando estudou a atratividade de iscas alimentares na captura de insetos em armadilhas Mcphail, a manga apresentou o cromatograma mais rico, entre todos os materiais analisados, tanto em número de compostos (22) quanto em intensidade de cada composto (elevadas contagens de área).

\begin{tabular}{|c|c|}
\hline \multicolumn{2}{|c|}{ Iscas } \\
\hline Mamão +Manga & $5,12 a$ \\
\hline Sardinha+Paçoca & $2,5 b$ \\
\hline \multicolumn{2}{|c|}{ Ambientes } \\
\hline Floresta Nativa & $3,87 a$ \\
\hline SAF & $3,75 \mathrm{a}$ \\
\hline \multicolumn{2}{|c|}{ Ordens de insetos } \\
\hline Coleoptera & $1,0 \mathrm{c}$ \\
\hline Diptera & $3,0 \mathrm{~b}$ \\
\hline Hymenoptera & $9,25 \mathrm{a}$ \\
\hline Orthoptera & $2 b c$ \\
\hline
\end{tabular}

Tabela 3. Número de insetos capturados nas iscas utilizadas, nos ambientes de Floresta Nativa e Sistema Agroflorestal e suas respectivas ordens, Rolim de Moura-RO. 2014

Medeiros (2009) analisou o perfil químico da manga e mamão e pode concluir que o perfil de voláteis da manga mostrou grande predominância em hidrocarbonetos terpênicos e aromáticos, sendo o 3 - careno o composto majoritário (quase 20 milhões de unidades 
de área). $\mathrm{O}$ atrativo à base de mamão tambem apresentou um perfil de voláteis composto por hidrocarbonetos terpênicos, porém, de compostos diferentes da manga: linalool e alguns de seus óxidos. O linalool corresponde a um feromônio e está envolvido com a atratividade sobre mariposas polinizadoras. Na floresta nativa a incidência da família Formicidae na isca de mamão com manga foi bem representativa em relação ao SAF, isso pode ter ocorrido devido a área ter um alto índice de preservação, propiciando um bom desenvolvimento da espécie no local.

O SAF não diferenciou da floresta nativa e das ordens de insetos capturados, a Hymenoptera foi a ordem mais recorrente, identificados pela família Formicidae. As formigas são como indicadores biológicos à perturbação, pois, a pressão antrópica provoca alterações na estrutura da comunidade (DELABIE et al., 2006), retratando indicação de alteração de habitats, destruição, contaminação, reabilitação, sucessão da vegetação, mudanças climáticas e consequentemente degradação dos solos e ecossistemas (McGEOCH, 1998).

Simões et al. (2009) testaram diferentes iscas na captura de insetos e, verificaram que a ordem de maior ocorrência foi a Hymenoptera $(68,79 \%)$ seguida das ordens Diptera $(21,2 \%)$, Lepidoptera $(1,11 \%)$, Blattodea $(6,04 \%)$, Orthoptera $(1,38 \%)$, Coleoptera $(0,54$ $\%)$, Heteroptera $(0,87 \%)$, Neuroptera $(0,15 \%)$, Homoptera $(0,09 \%)$. Os autores explicam a maior abundância de Hymenoptera ao fato de ter sido capturado um grande número de indivíduos da família Formicidae ( $89,0 \%$ dos Hymenoptera). A maioria dos insetos da ordem Hymenoptera foram atraídos por suco de maracujá indicando uma preferência por esse tipo de isca, isso se deve ao fato de necessitarem de alimentos mais energéticos (açúcar).

Costa et al. (2016), realizaram trabalho semelhante a este com iscas alimentares e os insetos com maior incidência nas armadilhas foram as formigas dos gêneros Atta e Solenopsis e as abelhas irapuás (Trigona spinipes) e os atrativos alimentares influênciaram a população desses insetos. Villar et al. (2010) realizaram trabalho de captura de insetos em pomar de citros utilizando armadilhas de garrafase, os atrativos de maior eficácia foram à base de goiaba, manga e laranja.

Para a interação entre iscas e ambientes (Tabela 4) percebe-se que $a$ isca a base de frutas foi a que atraiu maior quantidade de 
insetos na floresta nativa. No SAF não houve diferença significativa entre as iscas. Na Floresta nativa há maior ocorrência de Hymenoptera, família Formicidae, que são atraídas pelo açúcar, componente presente nas frutas, além destas serem constituídas por hidrocarbonetos terpênicos, aromáticos e feromônios (MEDEIROS, 2009).

\begin{tabular}{ccc}
\hline & \multicolumn{3}{c}{ Ambientes } \\
\cline { 2 - 3 } Iscas & Floresta Nativa & SAF \\
\hline Mamão +Manga & $6,25 \mathrm{aA}$ & $4,00 \mathrm{aB}$ \\
Sardinha+Paçoca & $1,5 \mathrm{bB}$ & $3,5 \mathrm{aA}$ \\
\hline DMS colunas e linhas & & 0,75 \\
\hline
\end{tabular}

Tabela 4. Interação entre iscas $x$ ambientes para a variável número de insetos capturados em Floresta Nativa e Sistema Agroflorestal, Rolim de Moura -RO. 2014

Para a interação entre iscas $x$ ordem de insetos (Tabela 5) percebe-se que para as ordens Coleoptera, Diptera e Orthoptera, não houve diferença de atratividade entre as iscas utilizadas. A isca a base de frutas foi mais atrativa para insetos da ordem Hymenoptera. Silva et al. (2014) quando avaliaram a entomofauna capturada em armadilha para dípteros na Reserva Biológica do Tinguá, afirmaram que a utilização de sardinha como isca pode atrair uma grande variedade de insetos e a presença de indivíduos da ordem Orthoptera na armadilha a base de sardinha não era esperada, já que esses insetos são amplamente conhecidos como sendo de hábito fitófago.

$O$ registro de ortópteros associados à matéria animal em decomposição realizado por Cruz e Vasconcelos (2006) refere-se a uma família diferente das encontradas no presente estudo, corroborando com os resultados deste trabalho.

\begin{tabular}{ccccc}
\hline & \multicolumn{4}{c}{ Ordens de Insetos } \\
\cline { 2 - 5 } Iscas & Coleoptera & Diptera & Hymenoptera & Orthoptera \\
Mamão +Manga & $1,0 \mathrm{aC}$ & $3,5 \mathrm{aB}$ & $14,5 \mathrm{aA}$ & $1,5 \mathrm{aC}$ \\
Sardinha+Paçoca & $1,0 \mathrm{aC}$ & $2,5 \mathrm{aB}$ & $4,0 \mathrm{bA}$ & $2,5 \mathrm{aB}$ \\
\hline DMS colunas & & \multicolumn{3}{c}{1,06} \\
\hline DMS linhas & & \multicolumn{3}{c}{1,43} \\
\hline
\end{tabular}

Tabela 5. Interação entre iscas x ordens de insetos para a variável número de insetos capturados Floresta Nativa e Sistema Agroflorestal, Rolim de Moura-RO. 2014 
Para a interação entre ambientes $\mathrm{x}$ ordens de insetos em relação à número de insetos capturados (Tabela 6), percebe-se que na floresta nativa e no SAF a ordem de maior ocorrência foi a Hymenoptera. Formigas são frequentemente utilizadas em estudos sobre diversidade e comunidades, por terem a vantagem de ser organismos dominantes nos ecossistemas, devido a sua importância tanto em riqueza de espécies quanto em número de indivíduos, além da relativa facilidade de coleta e da baixa mobilidade das populações (FOWLER et al., 1991).

\begin{tabular}{lcccc}
\hline & \multicolumn{4}{c}{ Ordens de Insetos } \\
\cline { 2 - 5 } Ambientes & Coleoptera & Diptera & Hymenoptera & Orthoptera \\
\hline Floresta Nativa & $1,5 \mathrm{aB}$ & $2,0 \mathrm{bB}$ & $11,0 \mathrm{aA}$ & $1,0 \mathrm{bB}$ \\
SAF & $0,5 \mathrm{aC}$ & $4,0 \mathrm{aB}$ & $7,5 \mathrm{bA}$ & $3,0 \mathrm{aB}$ \\
\hline DMS colunas & & \multicolumn{3}{c}{1,06} \\
DMS linhas & & \multicolumn{3}{c}{1,43} \\
\hline
\end{tabular}

Tabela 6. Interação entre ambientes $\mathrm{x}$ ordens de insetos para a variável número de insetos capturados em Floresta Nativa e Sistema Agroflorestal, Rolim de Moura-RO. 2014

A riqueza de espécies de insetos em alguns grupos taxonômicos pode aumentar se ocorrerem níveis intermediários de perturbações em florestas tropicais, como, por exemplo, aquelas perturbações parecidas com as naturais. No entanto, Thomazini e Thomazini (2000) alertaram que perturbações de maior grandeza, como desmatamentos e formação de fragmentos em florestas primárias, podem causar perda na riqueza e/ou diversidade de espécies de diversos grupos de insetos.

\section{CONSIDERAÇÕES FINAIS}

Em geral, a utilização da isca a base de frutas foi a mais eficiente para a captura e monitoramento de insetos em ambos ambientes, sendo a ordem Hymenoptera a de maior representatividade. Os ambientes de Floresta nativa e SAF não se diferenciaram quanto a presença da macrofauna capturada. 


\section{REFERÊNCIAS}

AGOSTI, D.; MAJER, J. D.; ALONSO, L.T.; SCHULTZ, T. editores. Ants: Standard methods for measuring and monitoring biodiversity. Washington: Smithsonian Institution Press; 2000. [cited 2010 Aug 1]. Available from: http://antbase.org/ databases/publications_files/publication-20330.htm.

ALVARES, C.A; STAPE, J.L.; SENTELHAS, P.C.; GONÇALVES, J.L.M.; SPAROVEK, G. Köpen's climate classification map for Brazil. Meteorologische Zeitschrift, Fast Track DOI: 10.1127/0941-2948/2013/0507. Gebru"der Borntraeger, Stuttgart, 2013.

BARROS, E.; MATHIEU, J.; TAPIA-CORAL, S.; NASCIMENTO, A. R. L.; LAVELLE, P. Soil macrofauna communities in Brazilian Amazonia. In: MOREIRA, F. M. S.; SIQUEIRA, J. O.; BRUSSAARD, L. (Ed.). Soil biodiversity in Amazonian and other Brazilian ecosystems. Wallingford: $C A B I, 2006$. p. 43-55.

BHARATI, T. E.; SATHIYANANDAM, V. K. R.; DAVID, P. M. M. Attractiveness of some food baits to the melon fruit fly Bactrocera cucurbitae (Coquillett) (Diptera: Tephritidae). International Journal of Tropical Insect Science, Saskatoon, SK, Canada, v.24, n.2, p. 125-134, 2004.

BROWN, G. G.; PASINI, A.; BENITO, N. P.; DE AQUINO, A. M.; CORREIA, M. E. F. Diversity and functional role of soil macrofauna comunities in Brazilian no-tillage agroecosystems. In: INTERNATIONAL SYMPOSIUM MANAGING BIODIVERSITY IN AGRICULTURAL ECOSYSTEMS, 2001, Montreal. Proceedings. Tokio: United Nations University, 2001. 1 CD-ROM.

BROWN, G. G.; MASCHIO, W.; FROUFE, L. C. M. Macrofauna do Solo em Sistemas Agroflorestais e Mata Atlântica em Regeneração nos Municípios de Barra do Turvo, SP, e Adrianópolis, PR. Colombo : Embrapa Florestas, Documentos 184, 51P. 2009.

CASTREJÓN-GÓMEZ, V. R.; ALUJA, M.; ARZUFFI, R.; VILLA, P. Two lowcost food attractants for capturing Toxotrypana curvicaudata (Diptera: Tephritidae) in the field. J. Econ. Entomol., v.97, n.2, p. 310-315, 2004.

CORNELIUS, M. L.; DUAN, J. J.; MESSING, R. H. Volatile host fruit odors as attractants for the oriental fruit fly (Diptera: Tephritidae). J. Econ. Entomol., v. 93, n.1, p. 93-100, 2000.

COSTA, A. C.; RAMOS, J. D. NASCIMENTO, D. P.; MIRANDA, J. M. S.; LAREDO, R. R. Armadilhas e iscas alimentares na captura de insetos na pitaia em Lavras-MG. Revista Cultivando o Saber. Volume 9 - n 3, p. 275 a 282, 2016.

CRUZ, T.M.; VASCONCELOS, S.D. Entomofauna de solo associada à decomposição de carcaça de suíno em um fragmento de Mata Atlântica de Pernambuco, Brasil. Biociências, 14(2):193-201, 2006.

DELABIE, J.H.C.; PAIM, V. R. L. M. NASCIMENTO, I. C.; CAMPIOLO, S.; MARIANO, C. S. F. As formigas como indicadores biológicos do impacto humano em manguezais da costa sudeste da Bahia. Neotrop. Entomol. vol. 35, n 5, 2006. 
FOWLER, H.G; DELABIE, J.H.C.; BRANDÃO,C.R.F.; FORTI, L.C.; VASCONCELOS, H.L. Ecologia nutricional de formigas. In: PANIZZI, A. R.; PARRA, J. R. P. (Org.). Ecologia nutricional de insetos e suas implicações no manejo de pragas. São Paulo: Manole, 1991. p. 131-223.

GARCIA-RAMIREZ, M. J.; CIBRIÁN-TOVAR, J.; ARZUFI-BARRERA, R.; LÓPEZ COLLADO, J.; SOTO-HERNANDEZ, M. Preferência de Anastrepha ludens (LOWE) (Diptera: Tephritidae) por volatiles de frutos verdes o amarillos de mango y naranja. Agrociencia, v.38, p. 423-430, 2004.

GRIMALDI, D. A.; ENGEL, M. The Evolution of Insects. Cambridge University Press, Cambridge. 755 p., 2005.

Instituto Brasileiro de Geografia e Estatística IBGE.Manual técnico da vegetação brasileira. Rio de Janeiro: IBGE. 92 p., 2012.

LEMOS, R. N. S.; SILVA, C. M. C.; ARAÚJO, J. R. G.; COSTA, L. J. M. P.; SALLES, J. R. J. Eficiência de substâncias atrativas na captura de moscas-dasfrutas (Diptera:Tephritidae) em goiabeiras no município de Itapecuru-mirim (MA). Revista Brasileira de Fruticultura, Jaboticabal-SP, v.24, n.3, dez. 2002.

LEWINSOHN, T. M.; PRADO, P. I. Quantas espécies há no Brasil? Megadiversidade 1(1):36-42, 2005.

MARIALVA, V. G. Diagnóstico Socioeconômico: JiParaná. Porto Velho: SEBRAE-RO. 76p. 1999.

McGEOCH M. A. The selection, testing and apllication of terrestrial insects as bioindicators. Biology Review, v. 73, p. 181-201, 1998.

MEDEIROS, M. A. A. Atratividade de iscas alimentares na captura de insetos em armadilhas McPhail. Mossoró, 2009. 101p. Tese (Doutorado) Universidade Federal Rural do Semi-Árido.

MONTES, S. M. N. M.; RAGA, A. Eficácia de atrativos para monitoramento de Ceratitis capitata (Diptera:Tephritidae) em pomar de citros. Arquivos do Instituto Biológico, São Paulo, v. 73, n. 3, p.317-323, 2006.

NAKANO, O.; SILVEIRA NETO, S.; ZUCCHI, R. A. Entomologia Econômica, Piracicaba-SP: Ceres, 314f.,1981.

OLIVEIRA, S. F.; DUTRA, C. P; BEIRAL, L. S.; MELO, P. V.; MELO, H. C.; LUCA, A. S. Eficiência de diferentes tipos de iscas na atração de formigas edáficas em trecho de mata no distrito de Fontanillas - Juína - MT. In: Il Jornada Científica do IFMT - Campus Juína. Desenvolvimento e Produção: A caminho da sustetabilidade, Anais... 2013.

RÉ. T. M. O uso de formigas como bioindicadores no monitoramento ambiental de revegetação de áreas mineradas. Tese (Doutorado) Escola Politécnica da Universidade de São Paulo, 244p., 2007.

RUPPERT, E.R.; FOX, R.S. \& BARNES, R.D. Zoologia dos invertebrados: uma abordagem funcional-evolutiva. São Paulo: Roca, 2005. 
SANTOS, M.S.; LOUZADA, J. N. C.; DIAS, N.; ZANETTI, R.; DELABIE, J. H. C.; NASCIMENTO, I. C. Riqueza de formigas (Hymenoptera, Formicidade) da serapilheira em fragmentos de floresta semidecídua da Mata Atlântica na região do Alto do Rio Grande, MG, Brasil. Iheringia, Série Zoologia 2006; 96(1): 95-101. http://dx.doi. org/10.1590/S0073-47212006000100017.

SALLES, L. A. Efeito do envelhecimento e da decomposição do atrativo na captura de adultos de Anastrepha fraterculus (Wied.) (Diptera:Tephritidae). Revista Brasileira de Agrociência, v.5, n.2, p. 147-148, 1999.

SILVA, A. B.; GADELHA, B. Q.; RIBEIRO, A. C.; FERRAZ, A. C. P.; AGUIAR, V. M. Entomofauna capturada em armadilha para dípteros na Reserva Biológica do Tinguá, Nova Iguaçu, Rio de Janeiro. Bioikos, Campinas, 28(1):11-23, jan./jun., 2014.

SILVEIRA, O.T.; ESPOSITO, M.C.; SANTOS, J.N. \&. GEMAQUE, F.E. Social wasps and bees captured in carrion traps in a rainforest in Brazil. Entomological Science, 8(1):33-9. 2005.

SIMÕES, M.H.; SILVA, M. S.; COSTA, F. A. F. Uso de iscas na captura de insetos. Anais do IX Congresso de Ecologia do Brasil. São Lourenço - MG, 2009.

SIPAM (Sistema de Proteção da Amazônia). Governo do Estado de Rondônia. Pedologia Rolim de Moura - RO. Porto Velho. 1 Mapa. Escala 1: 250.000. 2006.

PELENTIR, S. C. S. Eficiência de cinco modelos de armadilhas etanólicas na coleta de coleoptera: Scolytidae, em floresta nativa no município de Itaara-RS. Dissertação (Mestrado em Engenharia Florestal), Universidade Federal de Santa Maria (UFSM, RS), Santa Maria, 81p., 2007.

PEREIRA, C. C. Efeitos da ingestão de uma fonte protéica sobre a performance e seleção de dietas por fêmeas selvagens de Anastrepha obliqua (Diptera:Tephritidae). Tese (Doutorado), USP, Ribeirão Preto-SP, 74f., 2001.

THOMAZINI, M.J.; THOMAZINI, A.P.B.W. A fragmentação florestal e a diversidade de insetos nas florestas tropicais úmidas. Rio Branco: Embrapa Acre, 2000. 21p. (Embrapa Acre. Documentos, 57).

TRIPLEHORN, C. A.; JOHNSON, N. F. Borror and DeLong's introduction to study of insects. Farmington Hills: Brooks Cole Thomson, 2005.

VELOSO, V. R. S.; FERNANDES, P. M.; ROCHA, M. R.; QUEIROZ, M. V.; SILVA, R. M. R. Armadilha para monitoramento de controle das moscas-dasfrutas Anastrepha ssp. e Ceratitis capitata (Wied.) Anais da Sociedade Entomológica do Brasil, Londrina-PR, v.23, n.3, p. 487-493, 1994.

VILLAR, L.; CRUZ, M. C. M.; MOREIRA, R. A.; CURI, P. N. Atrativos alimentares na flutuação populacional de moscas-das-frutas e abelha irapuá. Scientia Agraria Paranaensis, v. 9, n. 3, p 67-73, 2010. 